\title{
Light Induced New Iminyl Radical Cyclization Reactions of Acyloximes to Isoquinolines
}

Rafael Alonso, Pedro J. Campos, Bárbara García and Miguel A. Rodríguez*

Departamento de Química, Universidad de La Rioja, Grupo de Síntesis Química de La Rioja, Unidad Asociada al C.S.I.C., Madre de Dios, 51, 26006 Logroño, Spain

\section{SUPPORTING INFORMATION}

TABLE OF CONTENTS
A. Experimental Procedures and Characterization Data for Compounds 1-17.
B. NMR Spectra for Compounds 1, 4, 6, 8, 10, 12, 14, 16 and 17. 


\section{General procedures}

${ }^{1} \mathrm{H}$ and ${ }^{13} \mathrm{C}$ NMR spectra were recorded on a Bruker ARX-300 spectrometer in $\mathrm{CDCl}_{3}$ with TMS as internal standard. Electrospray mass spectra were obtained on an HP 5989 B apparatus with an HP 59987 A interface, in positive-ion mode with methanol/water/acetic acid (60:35:5) as the mobile phase. UV spectra were recorded on an HP 8451A spectrophotometer. Elemental analyses were performed using a Model $1110 \mathrm{CE}$ instrument. Melting points were obtained on a Büchi SMP-20 apparatus and are uncorrected. All solvents were purified by standard procedures. Reagents were of commercial grades. 2-Phenyl- and 2vinylbenzaldehyde were prepared from $o$-anisaldehyde by the method reported in literature. ${ }^{1}$ 2-Ethynylbenzaldehyde was prepared from 2-bromobenzaldehyde using the method reported. ${ }^{2}$ 2-Phenyl- and 2-vinylbenzaldehyde were used for the preparation of acetophenone and benzophenone derivatives by addition of methyl or phenyl magnesium bromide followed by oxidation. $^{3}$

General Procedure for the Preparation of Oximes. A solution of the corresponding carbonyl compound $(10 \mathrm{mmol})$, hydroxylamine hydrochloride $(26 \mathrm{mmol})$ and pyridine (26 $\mathrm{mmol})$ in ethanol $(20 \mathrm{~mL})$ was heated under reflux for $14 \mathrm{~h}$. The solvent was then removed under vacuum, and the residue extracted with dichloromethane $(50 \mathrm{~mL})$. The organic layer was dried $\left(\mathrm{Na}_{2} \mathrm{SO}_{4}\right)$, filtered and evaporated under reduced pressure. The oxime was then ready to use.

General Procedure for the Preparation of Acyloximes. The oxime ( $5 \mathrm{mmol}$ ) was dissolved in $5 \mathrm{~mL}$ of pyridine. 1.3 equivalents of acetyl chloride were dropped, and the mixture was stirred for $2 \mathrm{~h}$ at room temperature. $50 \mathrm{~mL}$ of diethyl ether was then added and the resulting solution was washed with $\mathrm{HCl}\left(10 \%\right.$ aq solution) and $\mathrm{NaHCO}_{3}(5 \%$ aq solution). The organic layer was dried $\left(\mathrm{Na}_{2} \mathrm{SO}_{4}\right)$, filtered and evaporated under reduced pressure. The resulting acyloxime was purified by column chromatography (silica gel, hexane/EtOAc, 4:1).

2-Phenylbenzaldehyde $\boldsymbol{O}$-acetyloxime (1). White solid. Yield: $1076 \mathrm{mg}, 90 \%$. Mp: 81-83 ${ }^{\circ} \mathrm{C} .{ }^{1} \mathrm{H}$ NMR: $\delta 8.33(\mathrm{~s}, 1 \mathrm{H}), 8.14(\mathrm{~d}, 1 \mathrm{H}, J=6.6 \mathrm{~Hz}), 7.29-7.52(\mathrm{~m}, 8 \mathrm{H}), 2.18(\mathrm{~s}, 3 \mathrm{H})$ ppm. ${ }^{13} \mathrm{C}$ NMR: $\delta$ 155.38, 149.91, 131.43, 130.44, 129.90, 129.85, 128.65, 128.50, 128.03, $127.92,127.75,127.70,127.47,126.18,19.76$ ppm. GC-MS: $\mathrm{m} / \mathrm{z}: 239$. ES (+): m/z: $240(\mathrm{M}+$ 1). UV: $\lambda 239,258 \mathrm{~nm}\left(\varepsilon=11116,8197 \mathrm{M}^{-1} \mathrm{~cm}^{-1}\right)$. Anal. Calcd for $\mathrm{C}_{15} \mathrm{H}_{13} \mathrm{NO}_{2}: \mathrm{C}, 75.30 ; \mathrm{H}$, 5.48; N, 5.85. Found: C, 75.35; H, 5.46; N, 5.83.

Phenanthridine (2). Anal. Calcd for $\mathrm{C}_{13} \mathrm{H}_{9} \mathrm{~N}$ : C, 87.12; H, 5.06; N, 7.82. Found: C, 87.09; H, 5.07; N, 7.84.

2-Phenylbenzonitrile (3). Anal. Calcd for $\mathrm{C}_{13} \mathrm{H}_{9} \mathrm{~N}$ : C, 87.12; H, 5.06; N, 7.82. Found: C, 87.14; H, 5.05; N, 7.81.

2-Phenylacetophenone $\boldsymbol{O}$-acetyloxime (4). Yellow solid. Yield: $772 \mathrm{mg}, 61 \%$. Mp: 86-88 ${ }^{\circ} \mathrm{C} .{ }^{1} \mathrm{H}$ NMR: $\delta$ 7.45-7.52 (m, 2H), 7.34-7.41 (m, 7H), $2.20(\mathrm{~s}, 3 \mathrm{H}), 1.77(\mathrm{~s}, 3 \mathrm{H}) \mathrm{ppm}$. ${ }^{13} \mathrm{C}$ RMN: $\delta 168.91,166.47,140.54,140.49,135.13,130.23,129.78,129.53,128.88,128.53$,

\footnotetext{
${ }^{1}$ Cullen, K. E.; Sharp, J. T. J. Chem. Soc., Perkin Trans. 1 1993, 2961.

${ }^{2}$ Maeyama, K.; Iwasawa, N. J. Org. Chem. 1999, 64, 1344.

${ }^{3}$ Furniss, B. S.; Hannaford, A. J.; Smith, P. G. W.; Tatchell, A. R. Vogel's Textbook of Practical Organic

Chemistry, 5th ed.; Longman: Essex, U.K., 1989; pp 537, 609.
} 
127.56, 127.37, 19.73, 17.97 ppm. ES (+): m/z: $254(\mathrm{M}+1)$. UV: $\lambda 213 \mathrm{~nm}\left(\varepsilon=16867 \mathrm{M}^{-1} \mathrm{~cm}^{-}\right.$ ${ }^{1}$ ). Anal. Calcd for $\mathrm{C}_{16} \mathrm{H}_{15} \mathrm{NO}_{2}: \mathrm{C}, 75.87 ; \mathrm{H}, 5.97 ; \mathrm{N}$, 5.53. Found: $\mathrm{C}, 75.84 ; \mathrm{H}, 5.99 ; \mathrm{N}, 5.51$.

6-Methylphenanthridine (5). Anal. Calcd for $\mathrm{C}_{14} \mathrm{H}_{11} \mathrm{~N}$ : C, 87.01; H, 5.74; N, 7.25. Found: C, 86.98; H, 5.74; N, 7.28.

2-Phenylbenzophenone $\boldsymbol{O}$-acetyloxime (6). Yellow solid (obtained as a 60:40 mixture). Yield: $945 \mathrm{mg}, 60 \%$. Mp: $66-68{ }^{\circ} \mathrm{C} .{ }^{1} \mathrm{H}$ NMR: $\delta$ 7.08-7.53 $(\mathrm{m}, 13 \mathrm{H}), 6.94(\mathrm{~d}, 1 \mathrm{H}$, $J=6.5 \mathrm{~Hz}), 2.09(\mathrm{~s}, 1.2 \mathrm{H}), 1.97(\mathrm{~s}, 1.8 \mathrm{H}) \mathrm{ppm} .{ }^{13} \mathrm{C} \mathrm{RMN}: \delta 168.81,168.39,165.47,164.64$, $142.47,141.35,140.58,140.28,135.04,134.71,132.99,131.83,130.82,130.70,130.32$, $130.04,129.91,129.79,129.67,129.34,129.32$, 129.16, 128.84, 128.54, 128.26, 128.16, 127.86, 127.60, 127.31, 127.27, 127.02, 126.94, 19.73, 19.65 ppm. ES (+): m/z: $316(\mathrm{M}+1)$. $\mathrm{UV}: \lambda 211 \mathrm{~nm}\left(\varepsilon=27614 \mathrm{M}^{-1} \mathrm{~cm}^{-1}\right)$. Anal. Calcd for $\mathrm{C}_{21} \mathrm{H}_{17} \mathrm{NO}_{2}: \mathrm{C}, 79.98 ; \mathrm{H}, 5.43 ; \mathrm{N}, 4.44$. Found: C, 79.91; H, 5.45; N, 4.46 .

6-Phenylphenanthridine (7). Anal. Calcd for $\mathrm{C}_{19} \mathrm{H}_{13} \mathrm{~N}$ : C, 89.38; H, 5.13; N, 5.49. Found: C, 89.34; H, 5.15; N, 5.51.

2-Vinylbenzaldehyde $\boldsymbol{O}$-acetyloxime (8). Yellow oil. Yield: $775 \mathrm{mg}, 82 \% .{ }^{1} \mathrm{H}$ NMR: $\delta 8.69(\mathrm{~s}, 1 \mathrm{H}), 7.91(\mathrm{~d}, 1 \mathrm{H}, J=9 \mathrm{~Hz}), 7.42-7.51(\mathrm{~m}, 2 \mathrm{H}), 7.33\left(\mathrm{dt}, 1 \mathrm{H}, J_{1}=9 \mathrm{~Hz}, J_{2}=3 \mathrm{~Hz}\right), 7.05$ $\left(\mathrm{dd}, 1 \mathrm{H}, J_{1}=18 \mathrm{~Hz}, J_{2}=12 \mathrm{~Hz}\right), 5.63(\mathrm{~d}, 1 \mathrm{H}, J=18 \mathrm{~Hz}), 5.48(\mathrm{~d}, 1 \mathrm{H}, J=12 \mathrm{~Hz}), 2.25(\mathrm{~s}, 3 \mathrm{H}) \mathrm{ppm}$. ${ }^{13} \mathrm{C}$ RMN: $\delta 168.72,154.51,138.66,133.42,131.39,127.99,127.89,127.18,127.13,119.21$, $19.57 \mathrm{ppm}$. ES (+): m/z: $190(\mathrm{M}+1)$. UV: $\lambda 212,231,262 \mathrm{~nm}\left(\varepsilon=36865,30780,21832 \mathrm{M}^{-}\right.$ $\left.{ }^{1} \mathrm{~cm}^{-1}\right)$. Anal. Calcd for $\mathrm{C}_{11} \mathrm{H}_{11} \mathrm{NO}_{2}: \mathrm{C}, 69.83 ; \mathrm{H}, 5.86 ; \mathrm{N}, 7.40$. Found: $\mathrm{C}, 69.85 ; \mathrm{H}, 5.84 ; \mathrm{N}$, 7.37 .

Isoquinoline (9). Anal. Calcd for $\mathrm{C}_{9} \mathrm{H}_{7} \mathrm{~N}$ : $\mathrm{C}, 83.69 ; \mathrm{H}, 5.46 ; \mathrm{N}, 10.84$. Found: $\mathrm{C}$, 83.71; H, 5.48; N, 10.81 .

2-Vinylacetophenone $\boldsymbol{O}$-acetyloxime (10). Red oil. Yield: $700 \mathrm{mg}, 69 \%$. ${ }^{1} \mathrm{H}$ NMR: $\delta$ $7.56(\mathrm{~d}, 1 \mathrm{H}, J=6 \mathrm{~Hz}), 7.36-7.40(\mathrm{~m}, 1 \mathrm{H}), 7.29-7.31(\mathrm{~m}, 2 \mathrm{H}), 6.84\left(\mathrm{dd}, 1 \mathrm{H}, J_{1}=15 \mathrm{~Hz}, J_{2}=9 \mathrm{~Hz}\right)$, $5.70(\mathrm{~d}, 1 \mathrm{H}, J=15 \mathrm{~Hz}), 5.34(\mathrm{~d}, 1 \mathrm{H}, J=9 \mathrm{~Hz}), 2.32(\mathrm{~s}, 3 \mathrm{H}), 2.26(\mathrm{~s}, 3 \mathrm{H}) \mathrm{ppm} .{ }^{13} \mathrm{C} \mathrm{RMN}: \delta$ 168.89, 165.04, 136.12, 134.97, 134.41, 129.55, 128.53, 127.53, 126.26, 116.80, 19.75, 18.53 ppm. ES $(+): \mathrm{m} / \mathrm{z}: 204(\mathrm{M}+1)$. UV: $\lambda 212 \mathrm{~nm},\left(\varepsilon=25366 \mathrm{M}^{-1} \mathrm{~cm}^{-1}\right)$. Anal. Calcd for $\mathrm{C}_{12} \mathrm{H}_{13} \mathrm{NO}_{2}$ : C, 70.92; H, 6.45; N, 6.89. Found: C, 70.97; H, 6.46; N, 6.86 .

1-Methylisoquinoline (11). Anal. Calcd for $\mathrm{C}_{10} \mathrm{H}_{9} \mathrm{~N}$ : C, 83.88; H, 6.34; N, 9.78. Found: C, 83.91; H, 6.34; N, 9.75.

2-Vinylbenzophenone $\boldsymbol{O}$-acetyloxime (12). Yellow solid. Yield: $914 \mathrm{mg}, 69 \% .{ }^{1} \mathrm{H}$ NMR: $\delta 7.71(\mathrm{~d}, 1 \mathrm{H}, J=6 \mathrm{~Hz}), 7.59(\mathrm{~d}, 2 \mathrm{H}, J=7.5 \mathrm{~Hz}), 7.41-7.45(\mathrm{~m}, 2 \mathrm{H}), 7.32-7.37(\mathrm{~m}, 3 \mathrm{H})$, 7.09 (d, $1 \mathrm{H}, J=7.5 \mathrm{~Hz}), 6.54\left(\mathrm{dd}, 1 \mathrm{H}, J_{1}=15 \mathrm{~Hz}, J_{2}=9 \mathrm{~Hz}\right), 5.74(\mathrm{~d}, 1 \mathrm{H}, J=15 \mathrm{~Hz}), 5.21(\mathrm{~d}, 1 \mathrm{H}$, $J=9 \mathrm{~Hz}) 2.02(\mathrm{~s}, 3 \mathrm{H}) \mathrm{ppm} .{ }^{13} \mathrm{C}$ RMN: $\delta 168.48,164.05,135.32,134.05,133.84,131.87$, 131.01, 129.26, 128.46, 128.36, 127.81, 127.55, 124.99, 116.20, $19.57 \mathrm{ppm}$. ES (+): $\mathrm{m} / \mathrm{z}: 266$ $(\mathrm{M}+1)$. UV: $\lambda 214,249 \mathrm{~nm},\left(\varepsilon=54757,49623 \mathrm{M}^{-1} \mathrm{~cm}^{-1}\right)$. Anal. Calcd for $\mathrm{C}_{17} \mathrm{H}_{15} \mathrm{NO}_{2}$ : $\mathrm{C}$, 76.96; H, 5.70; N, 5.28. Found: C, 76.91; H, 5.72; N, 5.26.

1-Phenylisoquinoline (13). Anal. Calcd for $\mathrm{C}_{15} \mathrm{H}_{11} \mathrm{~N}$ : C, 87.77; H, 5.40; N, 6.82. Found: C, 87.75; H, 5.41; N, 6.84. 
2-Ethynylbenzaldehyde $\boldsymbol{O}$-acetyloxime (14). White solid. Yield: $701 \mathrm{mg}, 75 \%$. Mp: 80-82 ${ }^{\circ} \mathrm{C} .{ }^{1} \mathrm{H}$ NMR: $\delta 8.88(\mathrm{~s}, 1 \mathrm{H}), 8.09(\mathrm{~d}, 1 \mathrm{H}, J=9 \mathrm{~Hz}), 7.54(\mathrm{~d}, 1 \mathrm{H}, J=9 \mathrm{~Hz}), 7.39-7.45(\mathrm{~m}$, $2 \mathrm{H}), 3.45(\mathrm{~s}, 1 \mathrm{H}), 2.25(\mathrm{~s}, 3 \mathrm{H}) \mathrm{ppm} .{ }^{13} \mathrm{C} \mathrm{RMN}: \delta 168.82,154.37,133.20,131.35,129.35$, $126.55,125.29,123.40,83.74,82.99,19.71 \mathrm{ppm}$. UV: $\lambda$ 204, 230, 267nm, $(\varepsilon=69420,45892$, $16845 \mathrm{M}^{-1} \mathrm{~cm}^{-1}$ ). Anal. Calcd for $\mathrm{C}_{11} \mathrm{H}_{9} \mathrm{NO}_{2}: \mathrm{C}, 70.58 ; \mathrm{H}, 4.85 ; \mathrm{N}, 7.48$. Found: $\mathrm{C}, 70.61 ; \mathrm{H}$, $4.87 ; \mathrm{N}, 7.45$.

Benzophenone $\boldsymbol{O}$-acetyloxime (15). Anal. Calcd for $\mathrm{C}_{15} \mathrm{H}_{13} \mathrm{NO}_{2}$ : C, 75.30; $\mathrm{H}, 5.48$; N, 5.85. Found: C, 75.34; H, 5.46; N, 5.84.

Typical Procedure for the Irradiation of Acyloximes. The acyloxime $(0.6 \mathrm{mmol})$ was dissolved in $60 \mathrm{~mL}$ of the corresponding deoxygenated solvent and irradiated at room temperature under an Ar atmosphere, through Pyrex glass with a medium pressure-mercury lamp until the oxime was consumed (TLC, hexane/AcOEt, 4:1). The solvent was then removed with a rotary evaporator, and the products were separated by chromatography (silica gel, hexane/AcOEt). Irradiations of 1, 4, 6, and 14 were performed with a $400 \mathrm{~W}$ lamp, while 8, 10, and 12 were irradiated using a $125 \mathrm{~W}$ lamp. Irradiation of $\mathbf{1 5}$ was carried out in the presence of 10 equivalents of the corresponding alkyne with a $400 \mathrm{~W}$ lamp. Compounds 2, 3, $5,7,9,11$, and 13 were known.

1,3,4-Triphenylisoquinoline (16). Brown solid. Yield: $163 \mathrm{mg}, 76 \%$. Mp: 130-132 ${ }^{\circ} \mathrm{C} .{ }^{1} \mathrm{H}$ NMR: $\delta 8.17(\mathrm{~d}, 1 \mathrm{H}, J=3 \mathrm{~Hz}), 7.83(\mathrm{~d}, 1 \mathrm{H}, J=6 \mathrm{~Hz}), 7.71(\mathrm{~d}, 1 \mathrm{H}, J=3 \mathrm{~Hz}), 7.58-7.50(\mathrm{~m}$, $5 \mathrm{H}), 7.44-7.38(\mathrm{~m}, 5 \mathrm{H}), 7.32-7.29(\mathrm{~m}, 3 \mathrm{H}), 7.19-7.17(\mathrm{~m}, 3 \mathrm{H}) \mathrm{ppm} .{ }^{13} \mathrm{C} \mathrm{RMN}: \delta 159.75$, $149.59,140.84,139.77,137.50,136.92,131.30,130.40,130.18,129.88,129.71,128.26$, $127.95,127.95,127.49,127.46,127.23,126.93,126.52,125.98,125.38 \mathrm{ppm}$. ES (+): $\mathrm{m} / \mathrm{z}:$ $358(\mathrm{M}+1)$. Anal. Calcd for $\mathrm{C}_{27} \mathrm{H}_{19} \mathrm{~N}$ : C, 90.72; H, 5.36; N, 3.92. Found: C, 90.69; H, 5.38; $\mathrm{N}, 3.93$.

1-Phenylisoquinoline-3,4-dicarboxylic acid dimethyl ester (17). Yellow solid. Yield: $143 \mathrm{mg}, 74 \%$. Mp: 102-104 ${ }^{\circ} \mathrm{C} .{ }^{1} \mathrm{H}$ NMR: $\delta 8.18$ (d, $\left.1 \mathrm{H}, J=6 \mathrm{~Hz}\right), 8.02(\mathrm{~d}, 1 \mathrm{H}, J=9 \mathrm{~Hz})$, $7.84(\mathrm{t}, 1 \mathrm{H}, J=9 \mathrm{~Hz}), 7.73-7.68(\mathrm{~m}, 3 \mathrm{H}), 7.57-7.53(\mathrm{~m}, 3 \mathrm{H}) 4.12(\mathrm{~s}, 3 \mathrm{H}), 4.03(\mathrm{~s}, 3 \mathrm{H}) \mathrm{ppm} .{ }^{13} \mathrm{C}$ RMN: $\delta 168.24,166.14,162.26,138.49,138.15,133.80,131.78,130.28,129.75,129.45$, 128.67, 128.25, 127.75, 127.62, 125.68, 53.36, 53.30 ppm. ES (+): m/z: $322(\mathrm{M}+1)$. Anal. Calcd for $\mathrm{C}_{19} \mathrm{H}_{15} \mathrm{NO}_{4}$ : C, 71.02; H, 4.71; N, 4.36. Found: C, 70.96; H, 4.72; N, 4.38. 

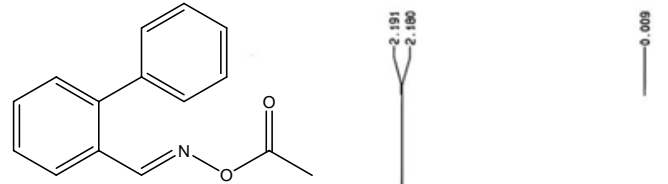

1
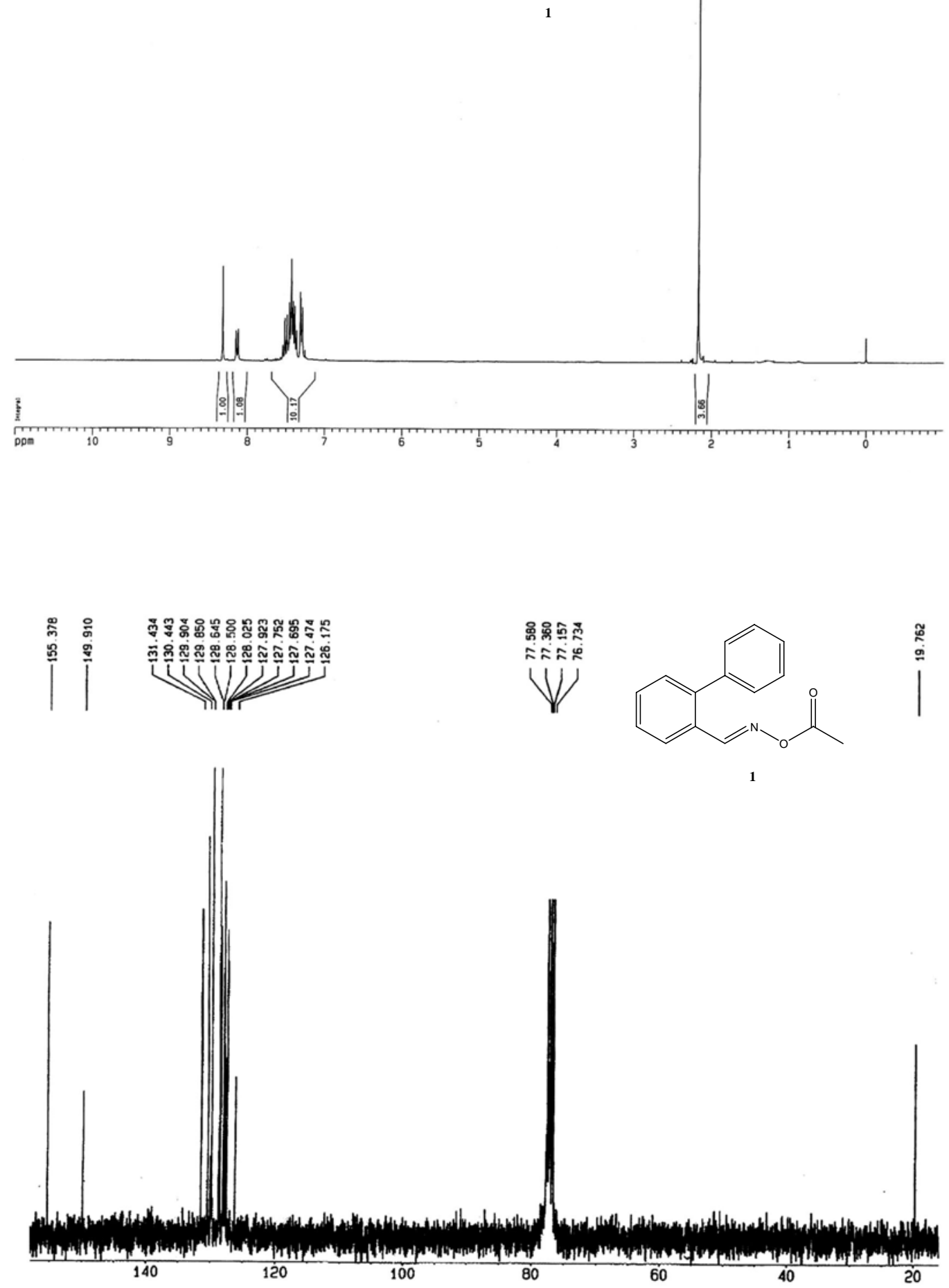

1 

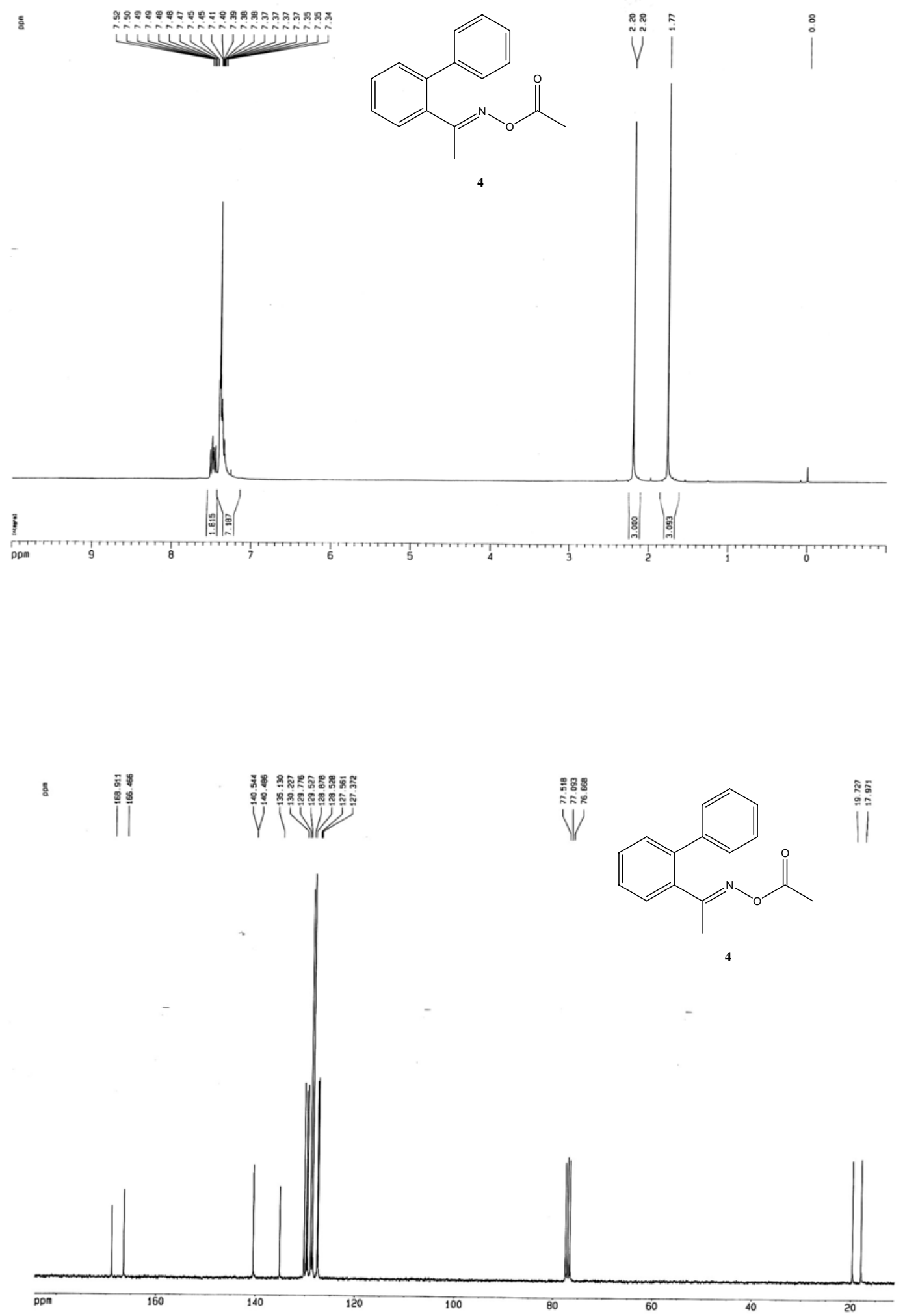


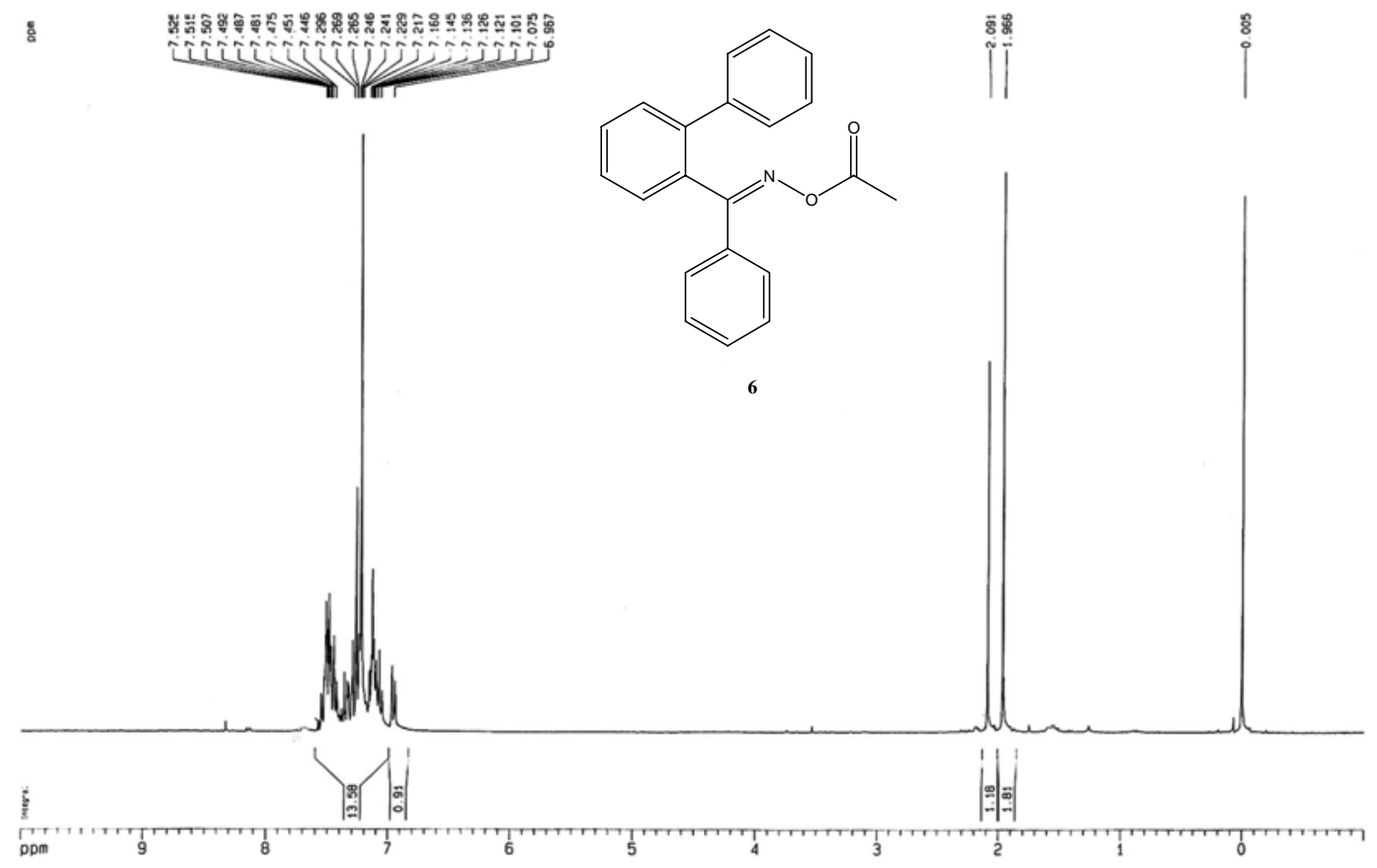

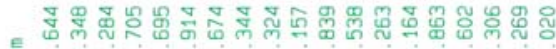

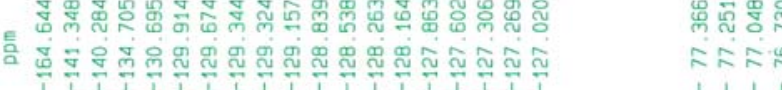

11

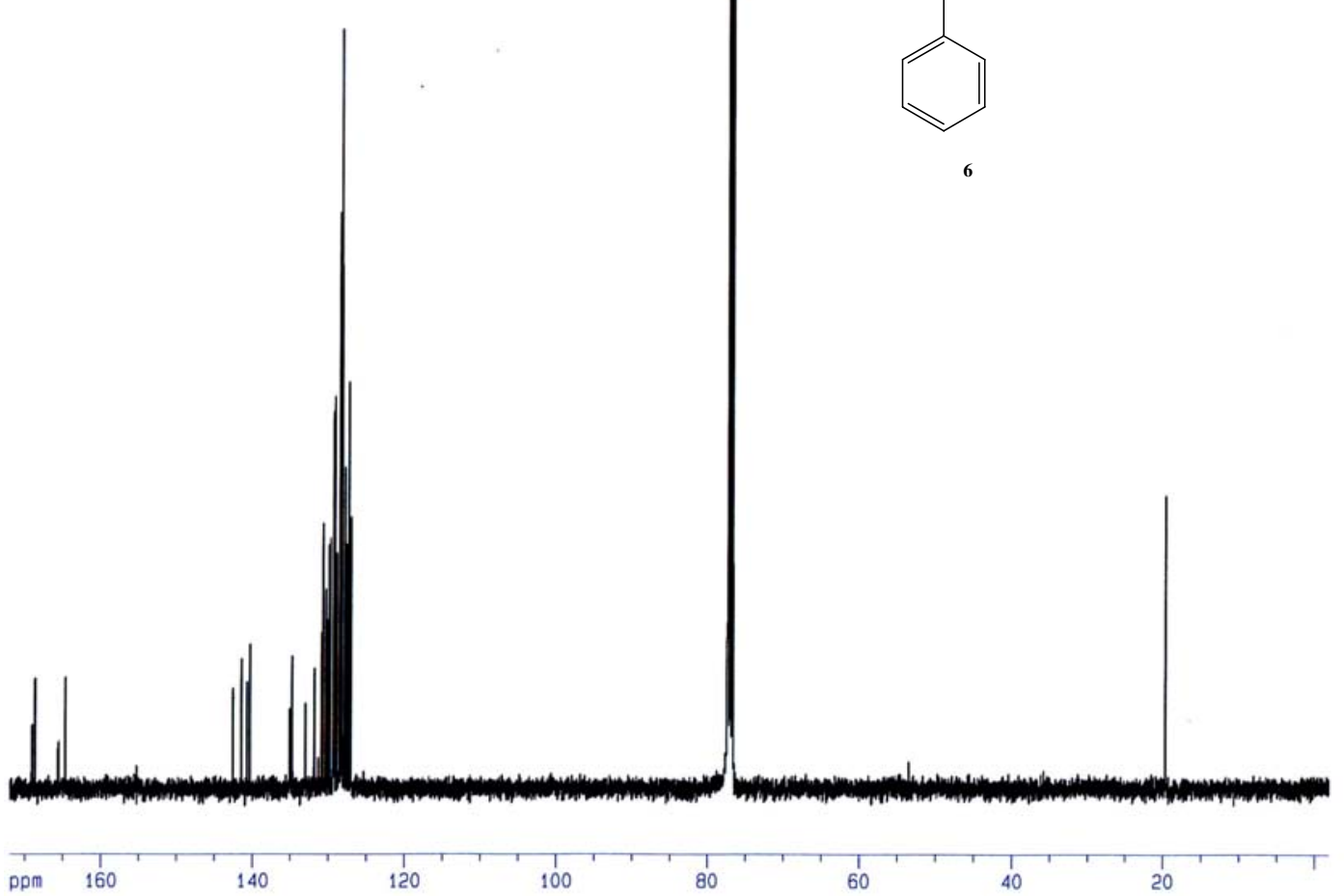




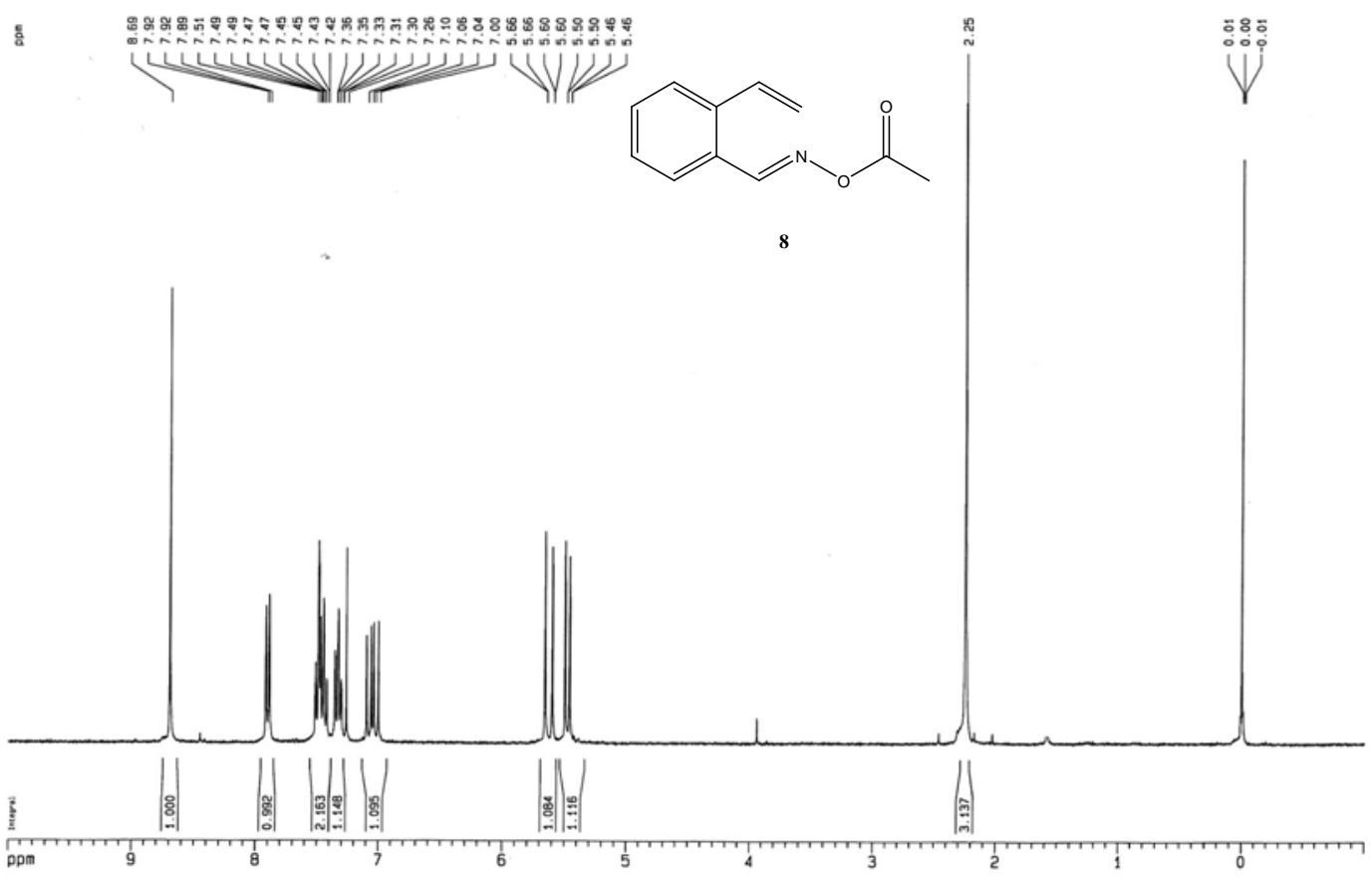

8
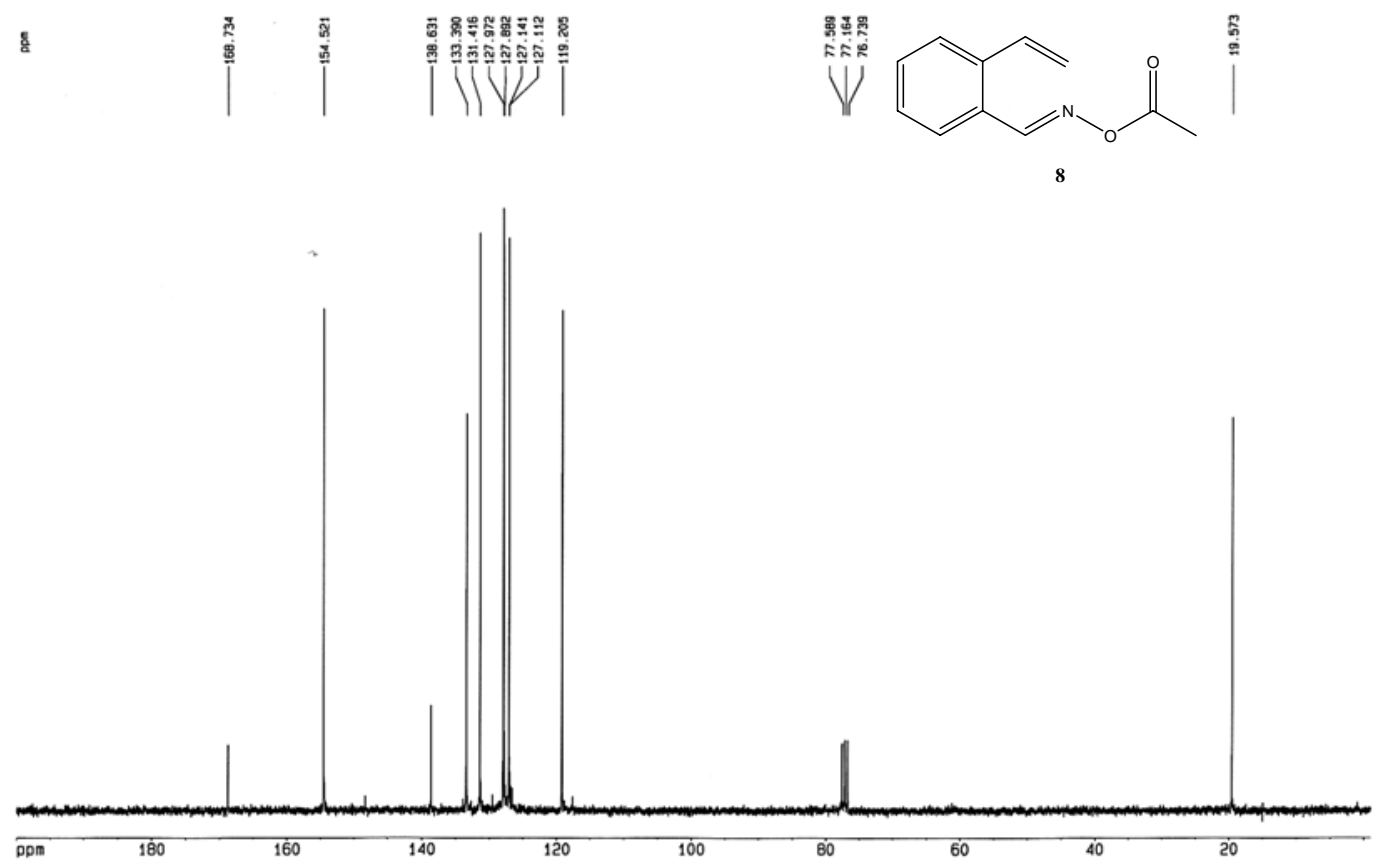
s

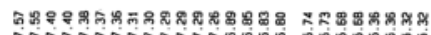
iiivi

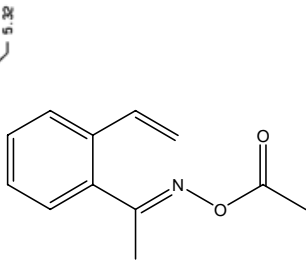

10
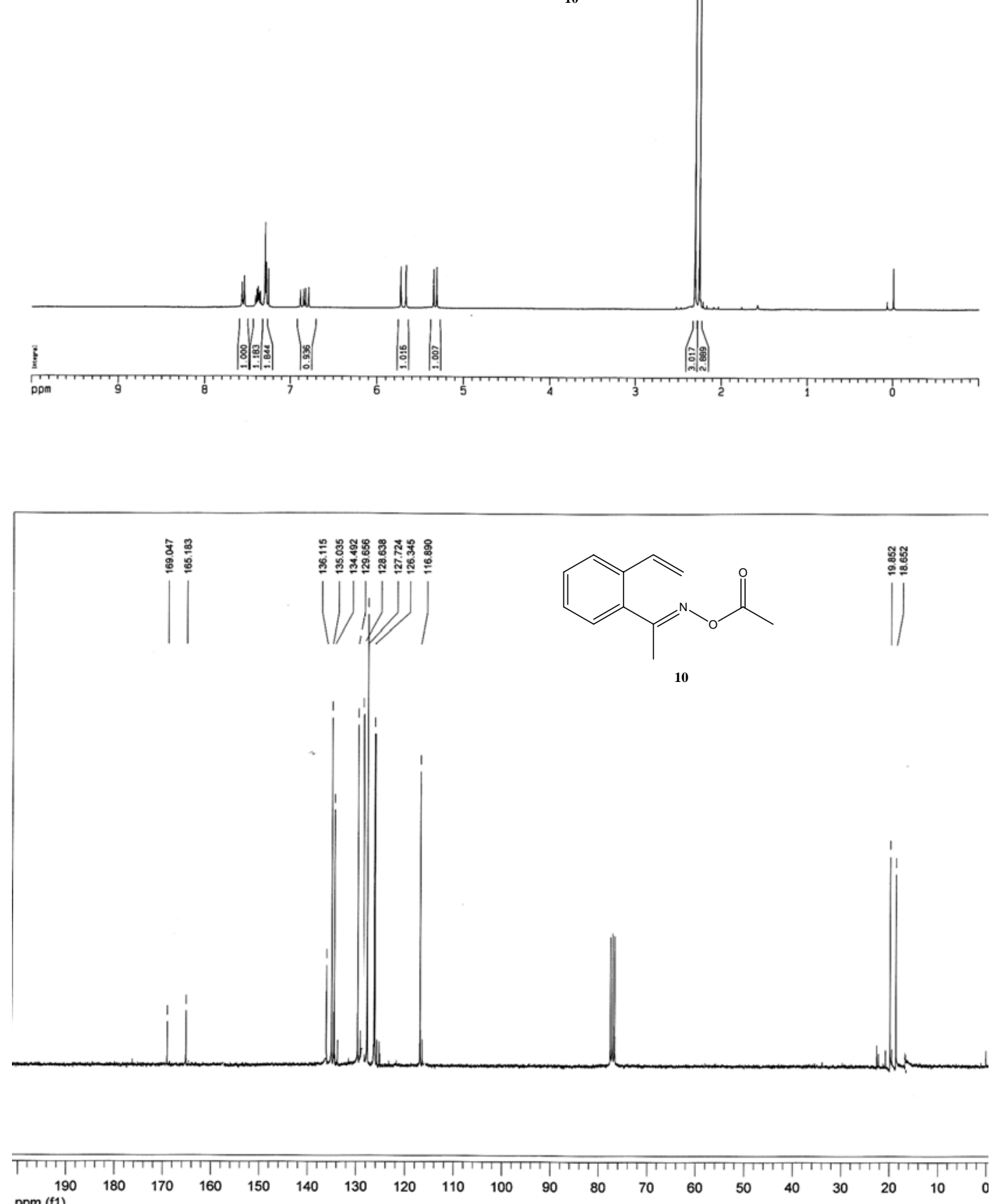

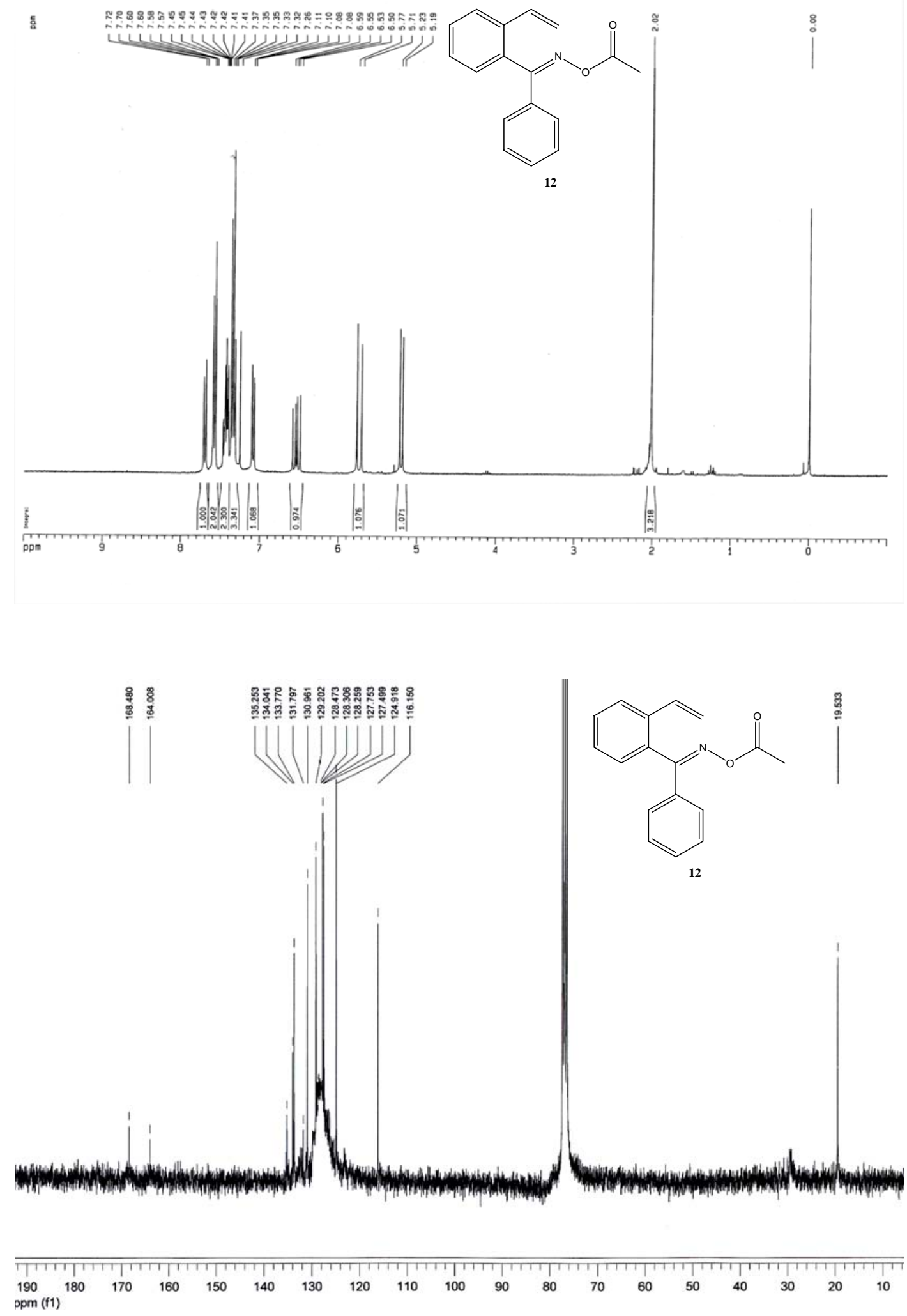


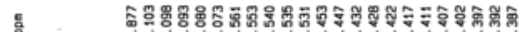

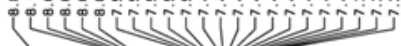<smiles>C#Cc1ccccc1/C=N/OC(C)=O</smiles>

14
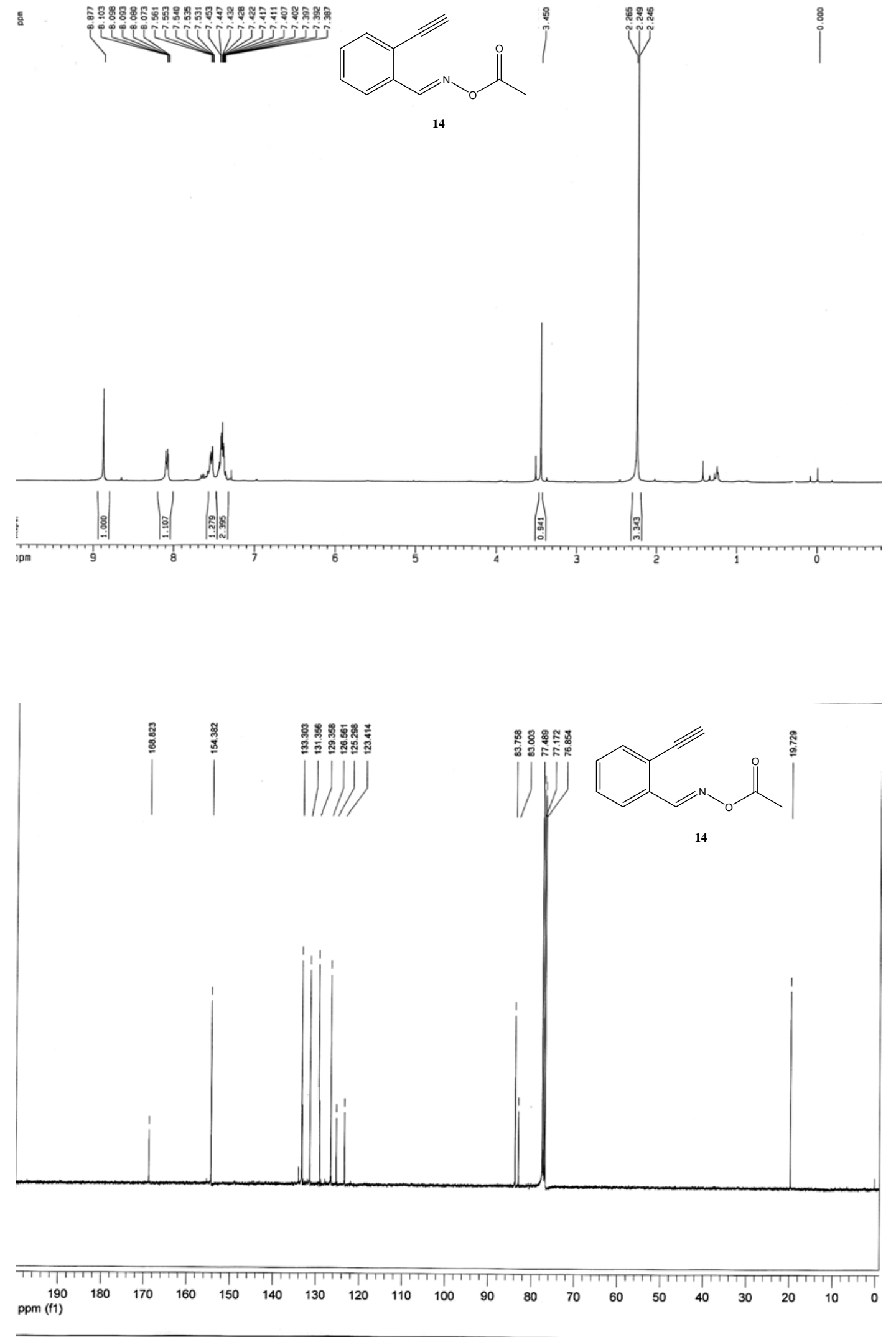


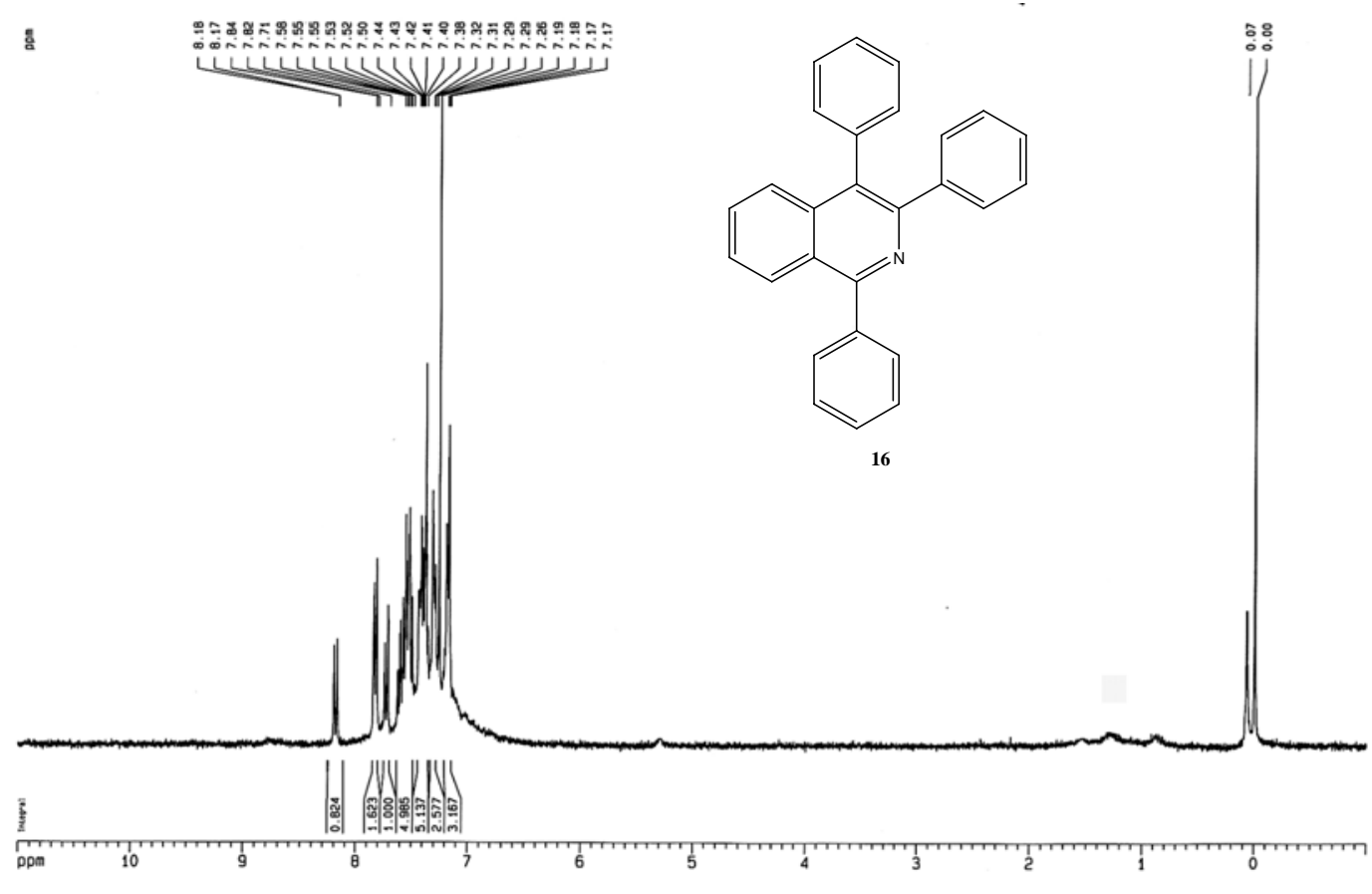

a

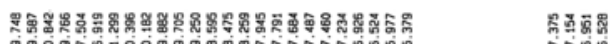

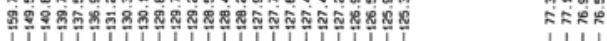
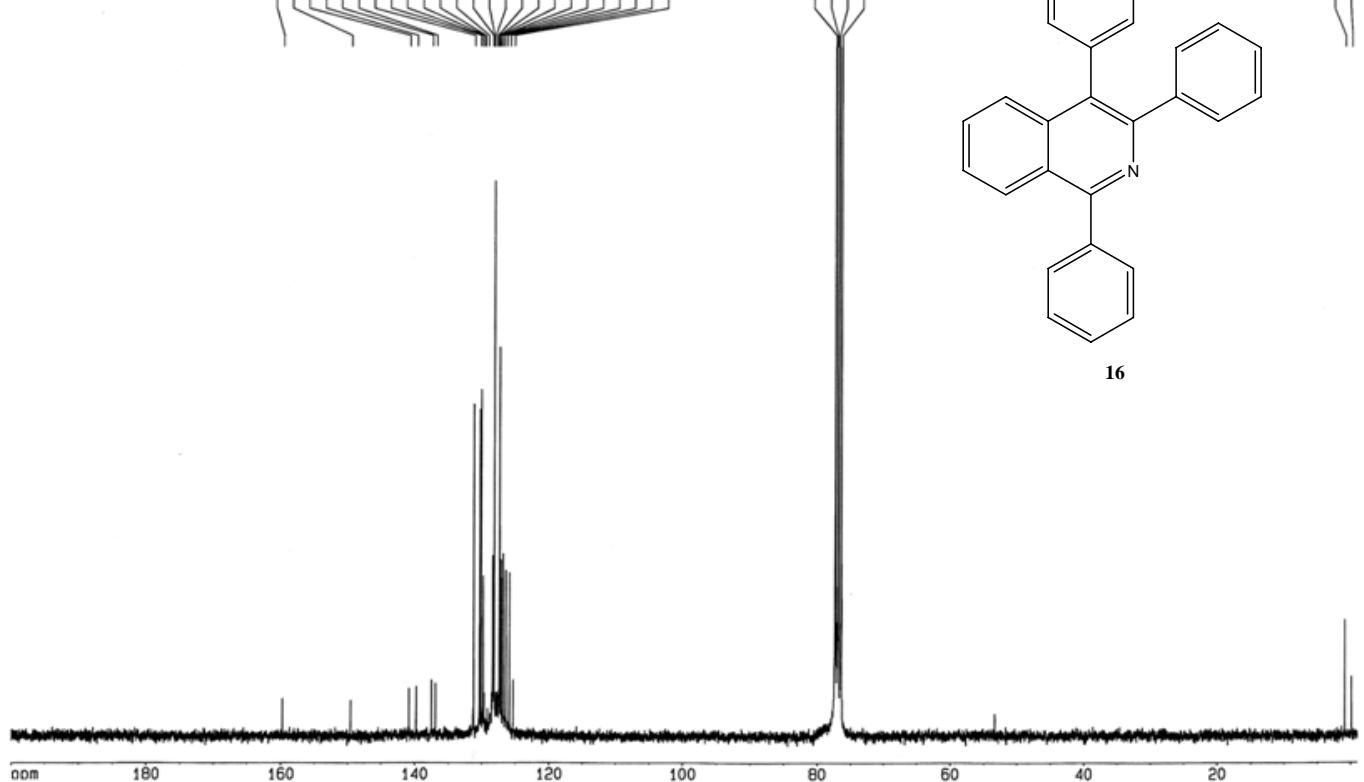
ता

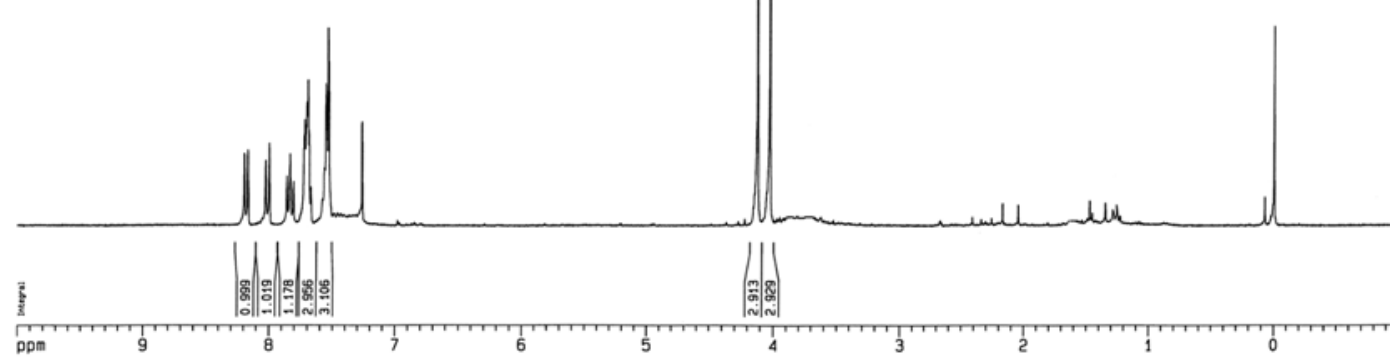

a

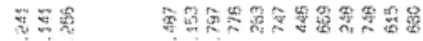

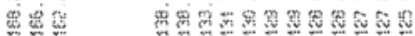

-

11
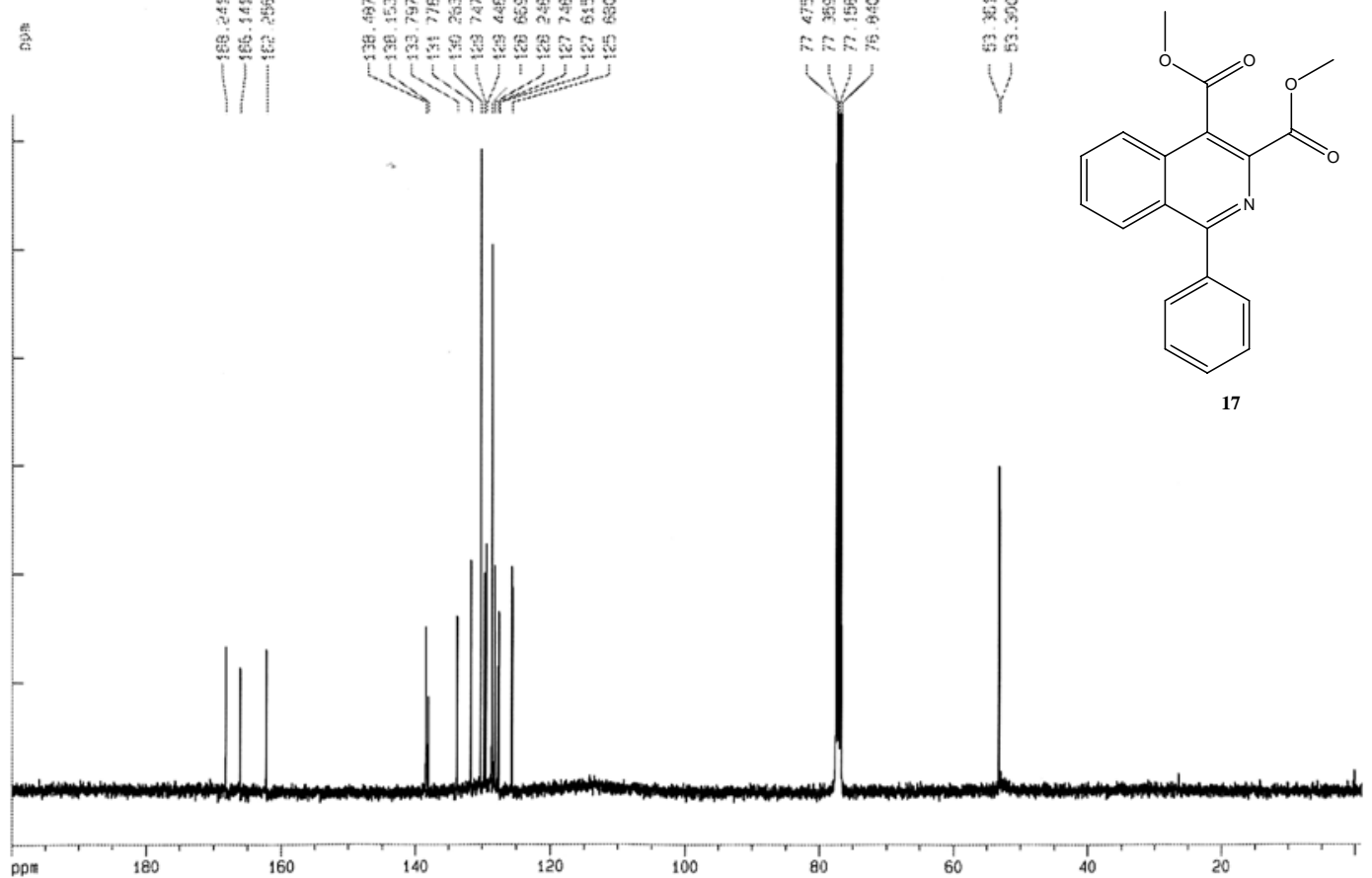<smiles>COC(=O)c1nc(-c2ccccc2)c2ccccc2c1C(=O)OC</smiles>

5ㅇํㅇ

IV<smiles>C=C/C(=C/C=C\C)c1cccc2ccccc12</smiles> 